\title{
Global convergence in leaf respiration from estimates of thermal acclimation across time and space
}

\author{
Mark C. Vanderwel ${ }^{1,2}$, Martijn Slot ${ }^{2,3}$, Jeremy W. Lichstein ${ }^{2}$, Peter B. Reich ${ }^{4,5}$, Jens Kattge ${ }^{6}$, Owen K. Atkin ${ }^{7}$, \\ Keith J. Bloomfield ${ }^{7}$, Mark G. Tjoelker ${ }^{5}$ and Kaoru Kitajima ${ }^{2,8}$
}

${ }^{1}$ Department of Biology, University of Regina, Regina, SK S4S 0A2, Canada; ${ }^{2}$ Department of Biology, University of Florida, Gainesville, FL 32611, USA; ${ }^{3}$ Smithsonian Tropical Research

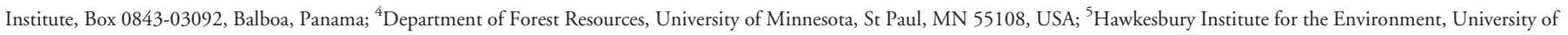

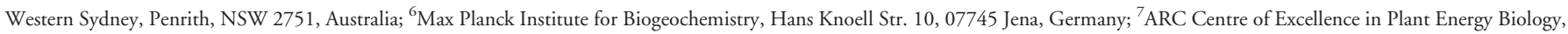
Research School of Biology, Australia National University, Building 134, Canberra, ACT 2601, Australia; ${ }^{8}$ Division of Forest and Biomaterial Science, Kyoto University, Kitashirakawa Oiwake-Cho, Kyoto 606-8502, Japan

Author for correspondence:
Mark C. Vanderwel
Tel: +1 3063372544
Email: mark.vanderwel@uregina.ca

Received: 19 January 2015

Accepted: 22 March 2015

New Phytologist (2015) 207: 1026-1037 doi: $10.1111 /$ nph.13417

Key words: autotrophic respiration, carbon flux, climate change, temperature, terrestrial biosphere modelling, thermal acclimation.

\section{Summary}

- Recent compilations of experimental and observational data have documented global temperature-dependent patterns of variation in leaf dark respiration $(R)$, but it remains unclear whether local adjustments in respiration over time (through thermal acclimation) are consistent with the patterns in $R$ found across geographical temperature gradients.

- We integrated results from two global empirical syntheses into a simple temperaturedependent respiration framework to compare the measured effects of respiration acclimationover-time and variation-across-space to one another, and to a null model in which acclimation is ignored. Using these models, we projected the influence of thermal acclimation on: seasonal variation in $R$; spatial variation in mean annual $R$ across a global temperature gradient; and future increases in $R$ under climate change.

- The measured strength of acclimation-over-time produces differences in annual $R$ across spatial temperature gradients that agree well with global variation-across-space. Our models further project that acclimation effects could potentially halve increases in $R$ (compared with the null model) as the climate warms over the 21st Century.

- Convergence in global temperature-dependent patterns of $R$ indicates that physiological adjustments arising from thermal acclimation are capable of explaining observed variation in leaf respiration at ambient growth temperatures across the globe.

\section{Introduction}

Autotrophic respiration (i.e. respiration by photosynthetic organisms) is a critical component of the global carbon budget, releasing about half of the carbon fixed annually by photosynthesis (Amthor \& Baldocchi, 2001; DeLucia et al., 2007; Zhang et al., 2009). As global temperatures are projected to rise over the $21 \mathrm{st}$ Century (IPCC, 2007), autotrophic respiration is expected to release a larger fraction of the $\mathrm{CO}_{2}$ fixed by photosynthesis in terrestrial ecosystems (Luo, 2007). Anticipated temperature-related increases in respiration could potentially contribute to a progressive weakening of the global terrestrial carbon sink over the 21 st Century (Friedlingstein et al., 2006).

A review by Smith \& Dukes (2013) on recent advances in modelling photosynthesis and respiration in terrestrial biosphere models (TBMs) highlighted two major challenges in quantifying temperature responses of autotrophic respiration. First, the biochemical processes controlling temperature-related variation in respiration rates are complex and poorly understood (Atkin \&
Tjoelker, 2003), and so predictions for how temperature affects respiration rely largely on empirically derived relationships. Second, many TBMs ignore differences between short- and longterm responses of respiration to temperature variation. Terrestrial biosphere models commonly assume that instantaneous response functions - for example, an exponential relationship between respiration and variation in temperature over minutes to hours can be applied to project respiration responses to temperature changes at seasonal and inter-annual time-scales. Although recent studies have begun to address these challenges (reviewed in Smith $\&$ Dukes, 2013), there remains considerable process uncertainty associated with model predictions of autotrophic respiration across time and space, particularly under new climatic conditions forecasted for the coming century.

Commonly used temperature response functions that are characterized by a doubling of respiration with each $10^{\circ} \mathrm{C}$ increase in temperature (i.e. $Q_{10}=2.0$ in Eqn 1 below) are inadequate for describing autotrophic respiration over either short or long timescales (Atkin \& Tjoelker, 2003; Atkin et al., 2005). One reason 
for this is that the instantaneous response is itself temperaturedependent, with respiration appearing to exhibit a weaker response to temperature at higher temperature ranges (Tjoelker et al., 2001). Further, individual plants exposed to colder or warmer temperatures over a period of days or weeks adjust their respiratory rates in a manner that partially compensates for shifts in ambient temperature (Lambers et al., 2008). This process is known as thermal acclimation. Acclimation is associated with decreases in the rate of respiration at a common, set measuring temperature when plants are exposed to high growth temperatures for extended periods (Atkin \& Tjoelker, 2003). Conversely, respiration rates at common temperature typically increase when plants acclimate to low growth temperatures. Acclimation may also affect the temperature sensitivity $\left(Q_{10}\right)$ or temperature optimum of respiration, although we do not consider these aspects further here.

Acclimation has been shown to occur as quickly as in just a few days (Bolstad et al., 2003; Lee et al., 2005), which suggests that changes in the temperature response function of respiration probably have a very strong influence on realized rates. Over extended time-scales (days, weeks or longer), thermal acclimation weakens the realized temperature sensitivity of respiration by lessening differences in respiration when plants experience sustained changes in ambient temperature. The concept of acclimation can also explain how identical genotypes differ in respiration rates when grown at different ambient temperatures, as is the case in controlled environments and common garden studies. If we assume that closely related plants growing in different sites are genetically similar, then differences in respiration at a common temperature may reflect acclimation effects (although genetic differences in $R$ could also be involved). Studies that have incorporated thermal acclimation of respiration into TBMs have concluded that this process has important effects on long-term carbon fluxes within biomes, although there is not a general agreement on its global effects (Wythers et al., 2005, 2013; King et al., 2006; Atkin et al., 2008; Chen \& Zhuang, 2013; Slot et al., 2014). Discrepancies among models may be attributable to the different ways in which acclimation is formulated, as well as to the varying approaches for deriving acclimation parameters from local data sources. The assimilation of data on variation in leaf respiration into TBMs is currently an active area of research (Atkin et al., 2014).

Quantitative estimates for the magnitude of thermal acclimation have been derived from experiments that measure the effects of recent growth temperature on leaf dark respiration $(R)$ in local plant populations. The designs of such experiments vary, but typically rely on either natural variation in ambient temperature, common gardens with different ambient temperatures, or experimental warming and control treatments. Each type of experiment allows the assessment of how $R$ acclimates to changes in temperature over a relatively short period. The degree of acclimation can be quantified by ratios of $R$ between acclimated and control plants, either at a common set temperature or at the plants' respective growth temperatures (Loveys et al., 2003). Slot \& Kitajima (2015) recently performed a meta-analysis of 43 experimental studies of acclimation in plants from all major biomes, summarizing the overall size of thermal acclimation effects and their main sources of variation. Their analysis showed that, when compared at a common measuring temperature, acclimation effects on leaf respiration were strongly related to the magnitude of temperature change, but did not vary significantly by biome, growth form, or other factors.

In addition to direct experiments, indirect evidence for adjustments in $R$ has been gleaned from comparative analyses of sites widely distributed across the globe. Databases such as Glopnet (Wright et al., 2004, 2006), TRY (Kattge et al., 2011), and GlobResp (Atkin et al., 2015) contain hundreds of records for $R$ across tropical, temperate, boreal and arctic biomes. Although these data lack the temporal resolution of experimental studies, they allow broad evaluations of how geographical patterns in respiration (which could result from both acclimation and long-term genetic adaptation) relate to climatic conditions. For example, in a comparison of $R$ at 20 different sites (mostly temperate forests and woodlands), Wright et al. (2006) found that respiration at mid-growing season temperatures increased with mean annual temperature, but that this increase was much less than would be expected from a simple instantaneous response to temperature. The partial adjustment of $R$ to ambient growth temperatures could reflect a combination of physiological acclimation, genetic adaptation, or variation in plant traits and species composition along climate gradients. To develop a more thorough understanding of global variation in $R$, Atkin et al. (2015) assembled data from 100 sites world-wide to describe relationships between $R$ and both climate variables and leaf traits. Their analysis shows that $R$ at ambient temperatures increases $c$. two-fold across a $20^{\circ} \mathrm{C}$ range in growth temperatures from the Arctic to the tropics.

The recent syntheses by Slot \& Kitajima (2015) and Atkin etal. (2015) provide a valuable opportunity to assess whether plants locally adjust their respiration over time (through phenotypic acclimation) in a manner that corresponds with how plants across large spatial gradients differ in $R$ with respect to temperature. In other words, is the change in $R$ from $10^{\circ} \mathrm{C}$ to $20^{\circ} \mathrm{C}$ over time for a plant at a given site equivalent to the difference in mean $R$ across sites with average temperatures of $10^{\circ} \mathrm{C}$ and $20^{\circ} \mathrm{C}$ ? We address this question using a simple temperature-dependent framework for leaf respiration through which the measured effects of respiration acclimation-over-time and variation-across-space can be compared to one another, and to a null model in which acclimation is ignored. We use this framework, with model parameters obtained directly from the recent data syntheses described above, to simulate respiration rates at locations across the globe over the course of a year. We then compare predictions of various acclimation algorithms in terms of: seasonal variation in $R$ at individual locations; spatial variation in mean annual $R$ across a global temperature gradient; and expected increases in global leaf-level $R$ rates under a representative climate change scenario. Output from these simple models illustrates how different formulations for thermal acclimation affect predicted global respiration patterns, and their future responses to climate warming. 


\section{Materials and Methods}

The following analysis has been designed to predict temperaturedependent respiration rates in mature, fully expanded leaves, which primarily reflect demands for respiratory products associated with cellular maintenance and potentially phloem loading. In addition to temperature, respiration rates are known also to vary with growth, tissue composition (e.g. $\mathrm{N}$ concentration), and functional traits (e.g. maximum carboxylation rate). Although these other factors are certainly important to respiration overall, they do necessarily influence the degree of acclimation or the temperature response of fully expanded leaves in a systematic way (Atkin et al., 2005, 2008).

We begin this section by describing the general form of instantaneous response and acclimation functions used in temperaturedependent respiration models. This is followed by descriptions of the model formulations that we have developed to incorporate the observed effects of acclimation-over-time and variationacross-space on $R$ predictions. Next, we describe how we obtained parameter values for the various acclimation models from two independent, global data sets of experimental and observational variation in $R$. Last, we describe our procedures for constructing scenarios to evaluate differences in $R$ across time and space under a set of different temperature-dependent models.

\section{Temperature response framework for respiration}

A common empirical framework for representing the instantaneous response of respiration to temperature uses the equation:

$R_{\mathrm{C}}=R_{\mathrm{REF}} \cdot Q_{10}^{\left(\frac{T_{\mathrm{C}}-T_{\mathrm{REF}}}{10}\right)}$,

Eqn 1

( $R_{\mathrm{C}}$, the respiration rate at a given current temperature; $T_{\mathrm{C}}$, the current temperature; $T_{\mathrm{REF}}$, an arbitrarily chosen reference temperature; $R_{\mathrm{REF}}$, the baseline respiration rate at $T_{\mathrm{REF}} ; Q_{10}$, a constant (or temperature-dependent function) that describes the proportional change in $R_{\mathrm{C}}$ per $10^{\circ} \mathrm{C}$ change in temperature.) Local studies that use this framework normally set $T_{\mathrm{REF}}$ to a constant that is representative of growth temperatures for the plant population being studied (e.g. 10,20 or $25^{\circ} \mathrm{C}$ ), whereas models (Collatz et al., 1991; Atkin et al., 2008) and comparative analyses (Reich et al., 1998; Wright et al., 2006) have often employed a single $T_{\mathrm{REF}}$ of $25^{\circ} \mathrm{C}$. The value $Q_{10}=2.0$ has been adopted widely in TBMs that use the $Q_{10}$ framework for autotrophic respiration (Raich etal., 1991; Aber \& Federer, 1992; Schimel et al., 1997). More recently, empirical observations of decreasing $Q_{10}$ with temperature (Tjoelker et al., 2001) have led to models that treat $Q_{10}$ as a temperature-dependent variable (Atkin \& Tjoelker, 2003; Atkin et al., 2005):

$Q_{10}=3.090-0.043 \cdot\left(\frac{T_{\mathrm{C}}+T_{\mathrm{REF}}}{2}\right)$.

Eqn 2

Incorporating this temperature sensitivity in the instantaneous response leads to lower respiration rates in future climate scenarios than are observed in TBMs that use the static value $Q_{10}=2.0$ (Wythers et al., 2005; King et al., 2006).

The framework above (Eqns 1 and 2) accounts for how the instantaneous response function changes across a biologically relevant temperature range. Acclimation responses can be accommodated in this framework by changing $R_{\mathrm{REF}}$ from a constant to a function of recent temperature history (Atkin \& Tjoelker, 2003). In TBMs that have incorporated acclimation responses, respiration in future climate scenarios is moderated even further than with the temperature-sensitive instantaneous responses described by Eqn 2 (Wythers et al., 2005; King et al., 2006).

\section{Alternative formulations of acclimation}

We developed a set of six simple leaf-level respiration models that incorporate acclimation-over-time and/or variation-across-space in different ways. The models are each variations within the temperature response framework described above (Eqns 1 and 2, including a temperature-dependent $Q_{10}$ ), and employ different representations for reference temperature $\left(T_{\mathrm{REF}}\right)$ and baseline respiration at $T_{\mathrm{REF}}\left(R_{\mathrm{REF}}\right)$ (Fig. 1 ; Table 1$)$. The general functional form for baseline respiration in each of the models is:

$\log _{10}\left(R_{\mathrm{REF}}\right)=r_{1}+r_{2} \cdot T_{\mathrm{REF}}+r_{3} \cdot\left(T_{\mathrm{A}}-T_{\mathrm{REF}}\right), \quad$ Eqn 3

( $T_{\mathrm{A}}$, an exponentially weighted average of temperatures over preceding days (defined in the Model Simulations section below) to which plants acclimate; $r_{1}-r_{3}$, empirical parameters from the syntheses by Slot \& Kitajima (2015) and Atkin etal. (2015) (detailed further in the Model Parameterization section below). Eqn 3 provides a convenient means to separate different timescales of thermal responses, and serves as an empirical framework for integrating thermal acclimation into model formulations of leaf-level respiration responses to temperature across both temporal and spatial scales. In the following paragraphs we describe the structure, rationale and assumptions of four of the different model formulations (another two models, which differ from those below only in their response to climate warming, are presented in the Model Simulations section).

Instant The simplest respiration model we consider, and which is similar to widely used formulations in current TBMs, calculates respiration rates at different temperatures using a fixed basal respiration rate at $25^{\circ} \mathrm{C}$, along with a temperature-dependent instantaneous response (Eqn 2) to predict respiration rates at other temperatures (Fig. 1a). This model assumes that temperature-related variation in respiration rates is unaffected by acclimation or other long-term processes. Unlike each of the following models, the basal respiration rate $\left(R_{25}\right)$ is a single constant value that applies across the globe.

Temporal This model modifies Instant by allowing for thermal acclimation processes to modulate $R$ rates at a standard reference temperature based on the temperature history over recent days. In this case, basal respiration rates $\left(R_{25}\right)$ are shifted up or down depending on whether temperatures over the preceding days have 


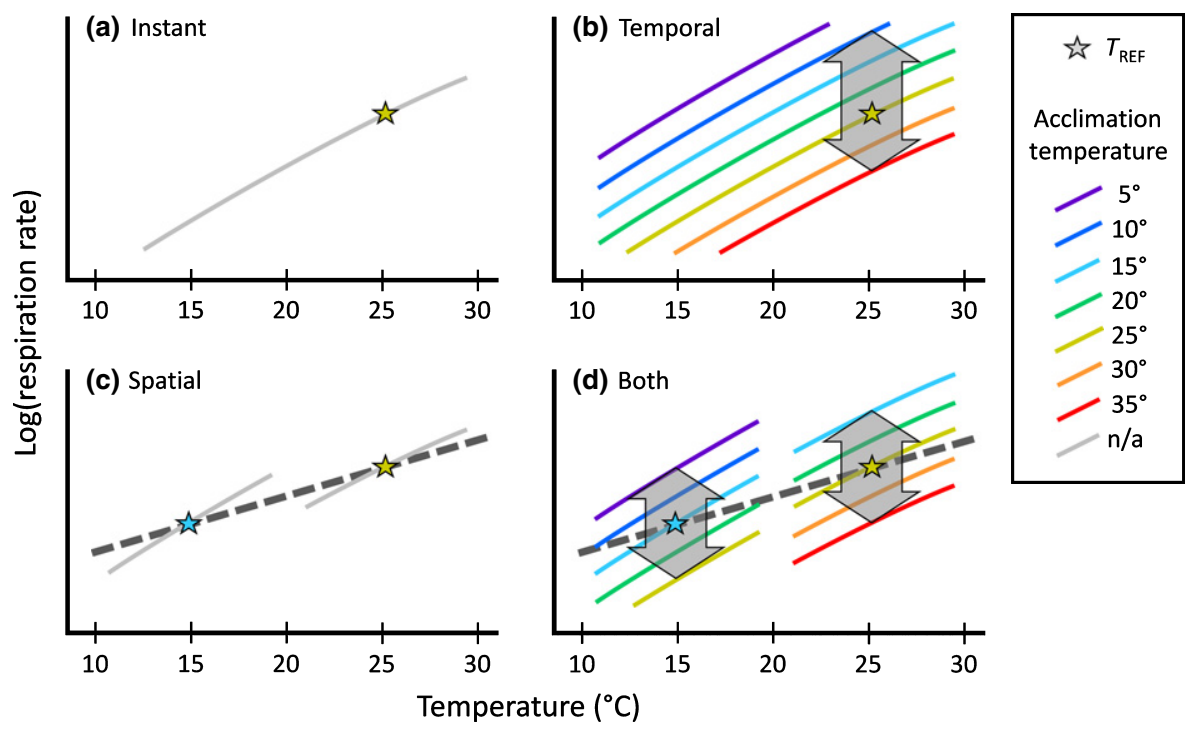

Fig. 1 Schematic illustration of temperature response curves under four alternative respiration models (Table 1). (a) In the most basic case, respiration rate at a given temperature is determined by applying the $Q_{10}$-based temperature response function from Eqn 2 (grey line) to the basal respiration rate ( $\left.R_{\mathrm{REF}}\right)$ at $25^{\circ} \mathrm{C}$ (yellow star). (b) Temporal acclimation changes respiration rates by shifting the temperature response function up or down depending on the acclimation temperature $\left(T_{\mathrm{A}}\right.$, different values of which are represented by the rainbow gradient of line colours) over preceding days. (c) To capture spatial variation in respiration at ambient growth temperatures across the globe, $T_{\mathrm{REF}}$ is set to the mean temperature of the warmest quarter (TWQ; hypothetical sites with TWQ $=15^{\circ} \mathrm{C}$ and $25^{\circ} \mathrm{C}$ represented by blue and yellow stars, respectively). The basal respiration rate at $T_{\text {REF }}$ is a log-linear function of TWQ (indicated by the thick dashed line), with respiration at a given temperature then determined by the temperature-dependent $Q_{10}$-based response from Eqn 2 (solid grey lines). (d) When both spatial variation and temporal acclimation are represented, acclimation shifts the temperature response up or down relative to the curve where $T_{\mathrm{A}}=\mathrm{TWQ}$, so that respiration responds separately to TWQ (moving along the thick dashed line), the acclimation temperature (moving up or down among the coloured lines), and the current temperature (moving along an individual coloured line). n/a, not applicable.

Table 1 Summary of temperature-dependent respiration model formulations and their ecophysiological interpretation

\begin{tabular}{|c|c|c|c|}
\hline Model label ${ }^{1}$ & $T_{\mathrm{REF}}^{2}$ & $\log _{10}\left(R_{\mathrm{REF}}\right)^{3}$ & Interpretation and notable characteristics \\
\hline Instant & $25^{\circ}$ & $r_{1}+r_{2} \times 25$ & $\begin{array}{l}\text { Respiration rates are defined by a constant basal rate }\left(R_{\mathrm{REF}}\right) \text { and a temperature-dependent, } Q_{10^{-}} \\
\text {based instantaneous response function. Acclimation processes are not represented. This } \\
\text { corresponds with the respiration formulations in many current terrestrial biosphere models, and is } \\
\text { used as a null model here for comparative purposes }\end{array}$ \\
\hline Temporal & $25^{\circ}$ & $r_{1}+r_{2} \times 25+r_{3} \times\left(T_{\mathrm{A}}-25\right)$ & $\begin{array}{l}\text { Incorporates thermal acclimation of baseline respiration based on the temperature history over } \\
\text { recent days. The structure of the model implies that thermal acclimation determines both } \\
\text { intra-annual variation in respiration over time and variation among different locations across the globe }\end{array}$ \\
\hline Spatial & $T W Q_{F I X}$ & $r_{1}+r_{2} \times \mathrm{TWQ}_{\mathrm{FIX}}$ & $\begin{array}{l}\text { Describes the manner in which respiration varies across the globe by defining baseline respiration } \\
\text { in relation to an ambient reference temperature (TWQ) at a given location. Like Instant, an } \\
\text { instantaneous response function }\left(Q_{10}\right) \text { describes variation in respiration at all time-scales }\end{array}$ \\
\hline Both & $\mathrm{TWQ}_{\mathrm{FIX}}$ & $\begin{array}{l}r_{1}+r_{2} \times \mathrm{TWQ}_{\mathrm{FIX}}+r_{3} \times \\
\quad\left(T_{\mathrm{A}}-\mathrm{TWQ}_{\mathrm{FIX}}\right)\end{array}$ & $\begin{array}{l}\text { Combines features of Temporal and Spatial to separately account for both spatial variation in } \\
\text { respiration and acclimation responses over time. Long-term changes are described by both } \\
\text { acclimation and instantaneous }\left(Q_{10}\right) \text { responses (because } T_{\mathrm{A}} \text { rises relative to } T W Q_{\mathrm{FIX}} \text { in future) }\end{array}$ \\
\hline Spatial-Equil & $T W Q_{D Y N}$ & $r_{1}+r_{2} \times \mathrm{TWQ}_{\mathrm{DYN}}$ & $\begin{array}{l}\text { Equivalent to Spatial under a reference climate, but implies that future warming-induced } \\
\text { respiration changes will mirror current spatial patterns across temperature gradients. The } \\
\text { instantaneous response }\left(Q_{10}\right) \text { controls intra-annual variation in respiration }\end{array}$ \\
\hline Both-Equil & $T W Q_{D Y N}$ & $\begin{array}{l}r_{1}+r_{2} \times \mathrm{TWQ}_{\mathrm{DYN}}+r_{3} \times \\
\quad\left(T_{\mathrm{A}}-\mathrm{TWQ}_{\mathrm{DYN}}\right)\end{array}$ & $\begin{array}{l}\text { Similar to Spatial-Equil, but with an added effect of acclimation to account for seasonal } \\
\text { temperature variation. The acclimation term does not reflect long-term changes in ambient } \\
\text { temperature because } T_{\mathrm{A}} \text { and } \mathrm{TW}_{\mathrm{DYN}} \text { are both presumed to increase in a warming climate }\end{array}$ \\
\hline
\end{tabular}

${ }^{1}$ All models share a common underlying framework where predicted respiration $\left(R_{\mathrm{C}}\right)$ at a given temperature $\left(T_{\mathrm{C}}\right)$ is modelled as $R_{\mathrm{C}}=R_{\mathrm{REF}} \times \mathrm{Q}_{10}^{\left(\left(T_{\mathrm{C}}-T_{\mathrm{REF}}\right) / 10\right)}$, $\mathrm{Q}_{10}=3.090-0.043 \times\left(\left(T_{\mathrm{C}}+T_{\mathrm{REF}}\right) / 2\right)$, and $R_{\mathrm{REF}}$ and $T_{\mathrm{REF}}$ are as specified in their respective columns.

${ }^{2} \mathrm{TWQ}_{\mathrm{FIX}}$, the temperature of the warmest quarter (TWQ) in a historic reference period (e.g., 1961-1990) that does not change through time; TWQ the temperature of the warmest quarter, either for the present or for a projected future climate.

${ }^{3} T_{\mathrm{A}}$, acclimation temperature, an exponentially weighted average temperature over recent days; $r_{1}, r_{2}, r_{3}$, model parameters derived from global empirical data in Atkin et al. (2015) and Slot \& Kitajima (2015).

been cooler or warmer than the reference temperature $\left(T_{\mathrm{REF}}\right)$ of $25^{\circ} \mathrm{C}$ (Fig. 1b). This model accommodates thermal acclimation of respiration to intra-annual variation in the ambient temperature at a particular location (e.g. summer versus winter). Importantly, when applied to multiple sites, the Temporal model also results in differences in leaf-level $R$ across spatial temperature 
gradients (e.g. tropical versus boreal biomes) through the same acclimation response to prior temperature that drives seasonal variation in $R$. Atkin et al. (2008) previously used this type of formulation to incorporate acclimation responses into the terrestrial biosphere model JULES.

Spatial This model explicitly accounts for leaf-level patterns of variation in respiration across space by allowing for spatially varying values of both $R_{\mathrm{REF}}$ and $T_{\mathrm{REF}}$ that are representative of ambient growing conditions. Following the approach outlined by Atkin et al. (2015), the Spatial model uses the mean temperature of the warmest quarter (TWQ; 1961-1990 reference period) in a given location as $T_{\mathrm{REF}}$. Basal respiration rate (i.e. respiration at $T_{\mathrm{REF}}$, with $T_{\mathrm{REF}}=\mathrm{TWQ}$ in this model) is then assumed to vary across space according to empirical patterns derived from a global respiration data set (Fig. 1c). Because observed differences in respiration rates across climate gradients tend to be smaller (for a given temperature difference) than those arising from short-term temperature variation, this model moderates variation in leaf-level respiration rates across space much as acclimation does in the Temporal model outlined above. In this case, site-to-site variation in $R$ could be underpinned either by phenotypic acclimation to the prevailing growth conditions or by genetic differences within and among species. Unlike the Temporal model, temperature responses in Spatial only describe variation across space, and do not account for seasonal patterns of acclimation over the course of a year. Yuan et al. (2011) used a similar formulation to redefine basal ecosystem respiration (analogous to $R_{\mathrm{REF}}$ ) across different locations as ecosystem respiration at the mean annual temperature. Their approach yielded improved predictions of monthly respiration compared with a globally constant basal respiration value.

Both. This model incorporates acclimation over time into the Spatial model. Like Spatial, TWQ is used as a reference temperature for a given location. Acclimation-over-time acts to adjust $R_{\mathrm{REF}}$ depending on whether recent temperatures have been cooler or warmer than TWQ (Fig. 1d). Compared with rates of $R$ predicted by Spatial, this formulation increases respiration at cooler times of the year (relative to the TWQ) and decreases it at warmer times of the year. Unlike the other models, Both explicitly separates out processes that modify $R$ across space from acclimation responses that act over time. Of the four models, Both provides the greatest flexibility in responses to spatial and temporal variation in temperature at multiple scales.

\section{Model parameterization}

Baseline respiration $\left(R_{\mathrm{REF}}\right)$ in all of our models was controlled by three parameters $\left(r_{1}-r_{3}\right.$ in Eqn 3). Parameters $r_{1}$ and $r_{2}$ describe how mass-based baseline respiration rates for single leaves depend on the long-term mean ambient temperature. We obtained the values $r_{1}=0.546$ and $r_{2}=0.016$ from the Atkin et al. (2015) global analysis of leaf dark respiration rates; these values represent the coefficients of a least-squared regression between $\log _{10}\left(R_{\mathrm{TWQ}}\right)$ and TWQ for 1113 species-site combinations of $R$ measurements in the GlobResp database, across all major biomes.
The relationship reported by Atkin et al. (2015) indicates that leaf-level respiration at the mean ambient temperature (TWQ) increases by c. $40 \%$ with each $10^{\circ} \mathrm{C}$ increase in TWQ across the globe, which is much less than expected from a simple instantaneous response (i.e. a $Q_{10}$ of c. 2).

Parameter $r_{3}$ describes the strength of thermal acclimation to ambient temperatures over recent days. A meta-analysis by Slot \& Kitajima (2015) showed that, at a given set temperature, acclimation effects on leaf-level respiration were strongly related to the magnitude of temperature change. Acclimation did not vary significantly by biome, growth form, leaf habit (deciduousness), or any of the other factors considered, however. Acclimation effects can be quantified by respiration ratios at a set temperature, defined as Acclim ${ }_{\text {SetTemp }}=R_{\text {REF, Control }} / R_{\text {REF, Acclim }}$ (Loveys et al. 2003). Here, we interpret control treatments as ones where $T_{\mathrm{A}}=T_{\mathrm{REF}}$. By using Eqn 3 and cancelling out all terms that are equal in the numerator and denominator, we obtain the expression $\operatorname{Acclim}_{\text {SetTemp }}=10^{\left(-r_{3} \cdot\left(T_{\mathrm{A}}-T_{\mathrm{REF}}\right)\right)}$. We then re-arrange this equation to estimate $r_{3}$ as:

$r_{3}=-\log _{10}\left(\operatorname{Acclim}_{\text {SetTemp }}\right) / \Delta T$,

Eqn 4

( $\Delta T$, the temperature change relative to a reference temperature.) Using lab- and field-based data from the Slot \& Kitajima (2015) meta-analysis, we obtained a global estimate of $r_{3}=-0.0158$ for thermal acclimation effects (Table 2). Our model variants that do not include acclimation-over-time (Instant, Spatial) effectively assume $r_{3}=0$.

\section{Model simulations}

We applied each of the models in Table 1 across a sample of the global land area under both reference and projected future temperature conditions. We first selected 500 random locations from across the global land surface. For each of these locations, we retrieved monthly mean temperatures and TWQ over the 19611990 reference period from the Worldclim database (Hijmans et al., 2005). To calculate respiration rates under reference conditions, we randomly selected for each location a single non-leap year between 1961 and 1990. For this single year per location, we obtained 6-hourly surface air temperature records (the shortest temporal resolution available) from the NCEP/NCAR reanalysis 1 climate data set (Kalnay et al., 1996). The 6-hourly temperature data were used with Eqns 1-3 to calculate respiration rates at each location four times daily over the selected reference year. The 6-hourly respiration rates were then averaged for the entire year to estimate an annual mean respiration rate. To quantify the effects of parameter uncertainty in our models, we also calculated annual mean respiration when parameters $r_{1}-r_{3}$ were varied by $\pm 2 \mathrm{SE}$. For simplicity, we assumed that respiration rates do not differ between day and night, other than through diurnal temperature changes.

In models that included acclimation-over-time, we used an exponentially weighted moving average to calculate acclimation temperature $\left(T_{\mathrm{A}}\right)$ as a recursive function of recent temperature history: 
Table 2 Summary of thermal acclimation effects from a meta-analysis of lab (growth cabinet) and field (common gardens, in situ warming, ambient temperature changes) studies across boreal, temperate and tropical biomes (from Slot \& Kitajima, 2015)

\begin{tabular}{|c|c|c|c|c|c|}
\hline Biome & $N_{\text {contrasts }}{ }^{1}$ & $N_{\text {species }}{ }^{2}$ & $\begin{array}{l}\text { Acclim }_{\text {SetTemp }}^{3} \\
(\text { mean } \pm S E)\end{array}$ & $\begin{array}{l}\Delta T^{4} \\
(\text { mean } \pm \mathrm{SE})\end{array}$ & $\begin{array}{l}\log _{10}\left(\text { Acclim }_{\text {SetTemp }}\right) / \Delta T \\
(\text { mean } \pm S E)\end{array}$ \\
\hline Boreal & 17 & 6 & $1.31 \pm 0.09$ & $7.0 \pm 0.91$ & $0.0145 \pm 0.0067$ \\
\hline Temperate & $156^{5}$ & 19 & $1.58 \pm 0.05$ & $11.2 \pm 0.52$ & $0.0161 \pm 0.0014$ \\
\hline Tropical & 13 & 13 & $1.16 \pm 0.06$ & $4.9 \pm 0.64$ & $0.0132 \pm 0.0035$ \\
\hline All & 186 & 33 & $1.53 \pm 0.04$ & $10.3 \pm 0.47$ & $0.0158 \pm 0.0014$ \\
\hline
\end{tabular}

${ }^{1}$ Number of temperature contrasts included.

${ }^{2}$ Number of species included.

${ }^{3}$ Acclimation ratio under the set-temperature method $\left(R_{\mathrm{REF}}\right.$, Control $/ R_{\mathrm{REF}}$, Acclim, where $R_{\mathrm{REF}}$, control and $R_{\mathrm{REF}}$, Acclim are the baseline respiration rates of control and acclimated plants, respectively).

${ }^{4}$ Temperature change applied to acclimated leaves.

${ }^{5}$ Excludes one outlier data point with only $0.3^{\circ} \mathrm{C}$ warming applied to Eucalpytus paucifloria.

$$
T_{\mathrm{A}, t+\Delta t}=0.1 \cdot T_{\mathrm{C}, t+\Delta t}+0.9 \cdot T_{\mathrm{A}, t},
$$

( $T_{\mathrm{A}, \mathrm{t}}$ the acclimation temperature at time $t, T_{\mathrm{C}, \mathrm{t}}$ the current temperature in the 6-hourly time series; $\Delta t, 6 \mathrm{~h} ; T_{\mathrm{A}, 0}=T_{\mathrm{C}, 0}$ (i.e. the temperature at 00:00 $\mathrm{h}$ on 1 January).) This formula allowed acclimation temperature to smoothly track the temperature history over previous days, with temperature weightings decreasing with elapsed time (e.g. temperatures on each of the past four days had total weights of $0.31,0.20,0.13,0.09$, etc., such that the past week's temperatures accounted for $95 \%$ of the exponentially weighted average temperature). This formulation is computationally efficient for modelling purposes, as it is calculated from a simple weighting of the current temperature and the immediately preceding acclimation temperature. The rate at which temperature weights decreased with time was somewhat faster than the duration of warming in the studies included by Slot \& Kitajima (2015) (median of $10 \mathrm{~d}$ for fully expanded mature leaves), although studies that have specifically monitored the time-scale of acclimation have found that adjustments in respiration occur within 2-3 d (Bolstad et al., 2003; Lee et al., 2005).

To project changes in respiration under future climate warming, we retrieved projections of monthly temperature anomalies, relative to the reference period, for the period 2070-2099. We used data from the UK Met Office Hadley Centre coupled model 3 (HADCM3; Gordon etal., 2000), whose projections are close to the mean of 23 general circulation models included in the IPCC AR4 synthesis report (IPCC, 2007). Data were obtained for an A1B emissions scenario, which, for this model, time period and set of sample locations, yielded average temperatures $4.5^{\circ} \mathrm{C}$ warmer than the reference period. We added the monthly temperature anomalies from the HADCM3 model to the 1-yr time series for each location to obtain a future time series with the same pattern of diurnal temperature fluctuations as the randomly selected year in the reference period. As before, 6-hourly respiration rates were averaged for the entire year to estimate an annual mean respiration rate under a warmer future climate. We again quantified uncertainty by varying model parameters by $\pm 2 \mathrm{SE}$.

In two of our respiration models (Spatial and Both), the reference temperature at a given location is specified by the TWQ. There are two possible ways to project future changes in respiration using these models. In the first case, we fix future reference temperatures to TWQ values from the reference period (1961-1990; 'static TWQ'). This formulation implies that, as the climate warms, changes in $R$ will be determined by short-term instantaneous responses (Spatial) and seasonal acclimation processes (Both). For example, if TWQ increases from 20 to $25^{\circ} \mathrm{C}$ at a particular site, the Spatial model would predict that $R_{\text {TWQ }}$ would increase by 46\% (from Eqns 1 and 2). In the second case, we update reference temperatures to projected TWQ values for the 2070-2099 period ('dynamic TWQ'). We designate this case by the model labels Spatial-Equil and Both-Equil to reflect the implicit assumption that respiration processes are, and will remain, in a dynamic equilibrium with climatic conditions at any given time. With this formulation, future changes in respiration rates over a 110 -yr period will mirror spatial differences that are currently found across global climate gradients. In the example above, the Spatial-Equil model would predict that a $5^{\circ} \mathrm{C}$ increase in TWQ would increase $R_{\mathrm{TWQ}}$ by $20 \%\left(=10^{\left.\left(r_{2} \cdot 5\right)\right)}\right.$, the same difference that is observed across a spatial gradient in TWQ (Atkin et al., 2015). The processes responsible for long-term changes in $R$ could include acclimation, genetic adaptation, changes in species composition, or biogeochemical feedbacks (Smith \& Dukes, 2013). It is not certain how much each of these processes contribute to differences in respiration rates, or how quickly they will respond to climate change. This scenario represents a useful basis for comparison with the first case (static TWQ), under the assumption that the key processes determining respiration rates keep pace with future climate warming.

\section{Results}

Incorporating direct estimates of thermal acclimation into our models changed respiration patterns in several ways. We illustrate these changes by examining three separate aspects of our projections in turn: seasonal variability in respiration rates at individual locations; spatial patterns in annual respiration rates across the globe; and global changes in respiration under future climate warming.

Models that incorporated acclimation-over-time based on Slot \& Kitajima's (2015) meta-analysis data (Temporal and Both) each moderated seasonal variation in respiration relative to the model 
that only accounted for variation-across-space (Spatial) (Fig. 2). In both acclimation-over-time models, respiration rates were higher during colder periods and lower during warmer periods in comparison to the Spatial model. The net result was a decrease in the intra-annual variance of respiration $(30 \%, 40 \%$ and $50 \%$ smaller coefficients of variation in sites with TWQ $=10.3,24.0$ and $31.3^{\circ} \mathrm{C}$, respectively), along with slightly higher mean annual values. Seasonal variation in respiration was very similar in the two models that included acclimation-over-time $(<2 \%$ difference in coefficient of variation between Temporal and Both), irrespective of how the models represented variation-across-space.

Acclimation-over-time yielded very similar changes in annual respiration rate across a global temperature gradient to variation-across-space, which was based on independent empirical observations from the GlobResp database (Atkin etal., 2015) (Fig. 3). The model with no acclimation (Instant) implied that, across the globe, a $10^{\circ} \mathrm{C}$ increase in mean annual temperature was associated with a near doubling (96\% increase) of mean annual respiration rates. Incorporating acclimation-over-time (Temporal) both increased respiration in locations with a mean annual temperature (MAT) less than c. $25^{\circ} \mathrm{C}$ and decreased respiration in regions with a higher MAT. These changes resulted in an overall increase in global respiration as well as lower spatial variability, with a $10^{\circ} \mathrm{C}$ increase in MAT now associated with only a $53 \%$ increase in respiration $\left(47-60 \%\right.$ when $r_{3}$ was varied by $\pm 2 \mathrm{SE}$ ). Importantly, the dampened increase in respiration with MAT mirrored that produced by both models derived from changes in observed ambient respiration with TWQ (Spatial and Both), where each $10^{\circ} \mathrm{C}$ increase in MAT increased respiration by $58 \%(52-65 \%$ when varying $r_{2}$ by $\pm 2 \mathrm{SE}$ ) and $53 \%(45-61 \%)$, respectively. The convergence of global variation in annual respiration between the Temporal, Spatial, and Both models implies that the direction and magnitude of thermal acclimation reported from local experimental studies are consistent with observed variation in ambient respiration (from GlobResp) across a global temperature gradient.

Projected respiration increases under climate warming were lessened by acclimation (Fig. 4). Models in which respiration responded to climate warming through short-term instantaneous responses (Instant and Spatial) projected a 34-36\% global increase in respiration rates between the 1961-1990 and 2070-2099 periods. Relative increases were greatest in cold regions $\left(51-52 \%\right.$ where TWQ $\left.<19^{\circ} \mathrm{C}\right)$ and lowest in hot regions $\left(28 \%\right.$ where TWQ $\left.>27^{\circ} \mathrm{C}\right)$. Acclimation-overtime moderated the projected increase in respiration by slightly more than half in the Temporal and Both models, with climate warming projected to increase global respiration by $16 \%$ in both cases. Again, relative increases were larger in cold regions compared with hot ones $(30 \%$ where TWQ $<19^{\circ} \mathrm{C} ; 11 \%$ where TWQ $>27^{\circ} \mathrm{C}$ ). The models in which we used ambient respiration across current spatial gradients to describe responses to climate warming (Spatial-Equil and Both-Equil) projected a $19-22 \%$ global increase in respiration. This change was considerably less than that produced by models that omitted acclimation-over-time (Instant and Spatial), but overlapped with the other models that included acclimation-over-time (Temporal and Both) when parameter uncertainty was considered. Unlike the other cases, the magnitude of warming-induced respiration increases in the two equilibrium models (Spatial-Equil and Both-Equil) was fairly consistent across the globe.

\section{Discussion}

We have used simple temperature-dependent models to scale up observational and experimental data on leaf respiration and project the effect of thermal acclimation on respiration rates across the globe. We show that the measured strength

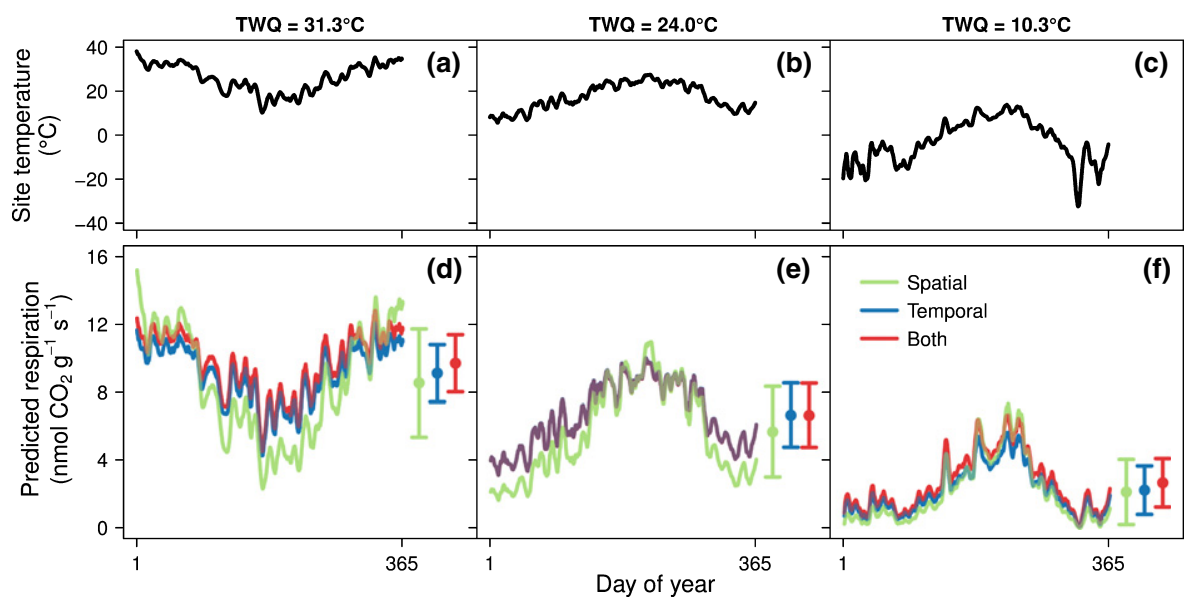

Fig. 2 Intra-annual variation in predicted respiration for individual sites representing the 90th, 50th and 10th percentiles of mean temperature of the warmest quarter (TWQ) under the three acclimation models illustrated in Fig. 1(b-d). The time series for site temperature (a, b, c) and predicted respiration rate $(d, e, f)$ are each smoothed to 1-wk running means. The error bars at the right of each bottom panel show the annual mean and SD of respiration under each model. The Temporal acclimation model moderates respiration at both warm and cold parts of the year compared with the Spatial model, resulting in lower intra-annual variation. The model labelled Both, in which spatial and temporal variations in respiration are regulated independently of one another, yields predictions close to those produced by the Temporal model (producing overlapping lines that appear purple in (e)). 


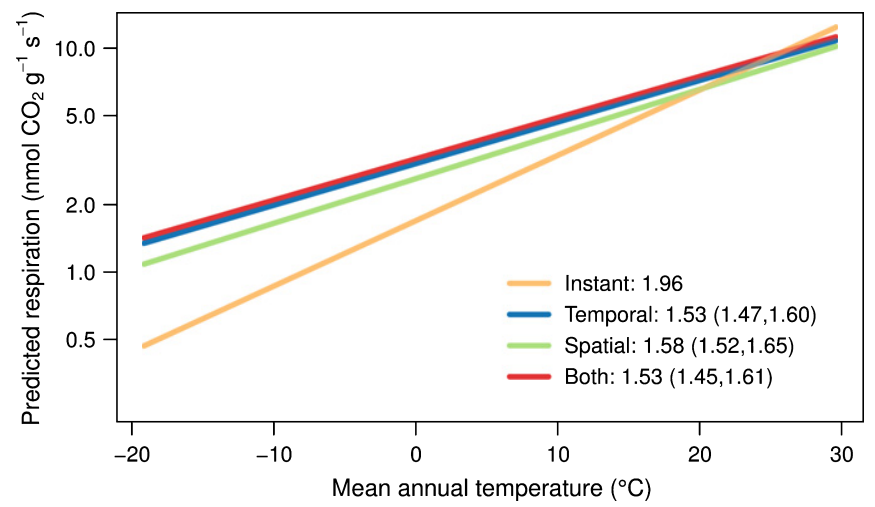

Fig. 3 Exponential relationships between predicted mass-based annual mean respiration rates and mean annual temperature (MAT) for the four respiration models illustrated in Fig. 1. Each line represents the fit of an exponential regression to model predictions for annual mean respiration at 500 sites across the globe $\left(R^{2}=0.96-0.99 ; \mathrm{RMSE}=0.29-0.54\right)$. The numbers beside each model label indicate the proportional change in annual respiration per $10^{\circ} \mathrm{C}$ increase in MAT, followed in parentheses by the range when acclimation parameters were varied by \pm 2 SE.

of acclimation-over-time (derived from a recent meta-analysis by Slot \& Kitajima (2015)) produces differences in annual respiration across spatial temperature gradients that agree well (within limits of parameter uncertainty) with global empirical patterns of variation-across-space from an independent data set (Atkin et al., 2015) (Fig. 3). Further, our simple models project that acclimation can greatly moderate future temperature-related increases in leaf-level dark respiration rates as the climate warms over the 21st Century (Fig. 4). These results contribute to a growing body of literature that highlights the importance of thermal acclimation of respiration to the global terrestrial carbon cycle (Wythers et al., 2005, 2013; King etal., 2006; Atkin etal., 2008; Chen \& Zhuang, 2013; Slot et al., 2014).

While previous studies (such as those cited directly above) have incorporated acclimation into sophisticated TBMs, our approach rests on simple conceptual formulations for the temperature dependence of respiration. These model forms have the advantages of being readily parameterized from published data, and of enabling a close analysis of the structure of the models themselves (i.e. the particular formulations of $T_{\mathrm{REF}}$ and $R_{\mathrm{REF}}$ in Eqns 1-3; Table 1). Our simple models also have limitations; for example, we do not attempt to represent growth (or growth respiration) responses to temperature variation, nor do we attempt to extrapolate from leaf-level respiration to other plant tissues or ecosystem-level fluxes. It is expected that total global leaf area will increase under climate change (Betts et al., 1997; Li et al., 2014), which would increase total leaf and autotrophic respiration (as well as photosynthesis) independently from changes that occur at the leaf level. Also, our climate simulations assume that daytime and nighttime temperatures will change by the same amount, but many projections indicate that daily minimum temperatures will increase more than daily maximum temperatures (IPCC, 2007). Given these types of simplifications, it would be inappropriate to formally compare predictions from our models to the quantitative effects of acclimation that have been estimated by TBMs. Nevertheless, our simple models occupy an important and largely unexplored space because, unlike TBMs, they are closely tied to global compilations of both data and results from empirical studies of respiration acclimation.

\section{Comparing acclimation effects across time to spatial variation}

As expected, acclimation-over-time moderated variation in respiration rates over the course of a year (Fig. 2). By increasing respiration in cold seasons and decreasing it in warm ones, acclimation (as represented in the Temporal model) produced less intra-annual variation in respiration than in the Spatial model (which captures the potential effects of acclimation across space, but not time). This finding is a useful proof-of-concept, and supports empirical studies (Tjoelker et al., 2008, 2009) showing that seasonal variation in respiration is dampened by acclimation processes. We also found that, when spatial and temporal patterns of acclimation are both accounted for in a single model (Both), intra-annual variation is nearly equivalent to that in the model based on acclimation-over-time alone (Temporal). In each of these two models, the magnitude of intra-annual variation is
Fig. 4 Predicted increases in annual mean mass-based respiration rates from 19611990 to $2070-2099$ in a projected A1B climate scenario in which the mean temperature increases by $4.5^{\circ} \mathrm{C}$ between these periods. Error bars show the range of predicted increase when acclimation parameters were varied by $\pm 2 \mathrm{SE}$. (not applicable for the Instant model, and a negligible effect in the Spatial model). The labels for each panel refer to different ranges of mean temperature of the warmest quarter (TWQ) in the 1961-1990 period.
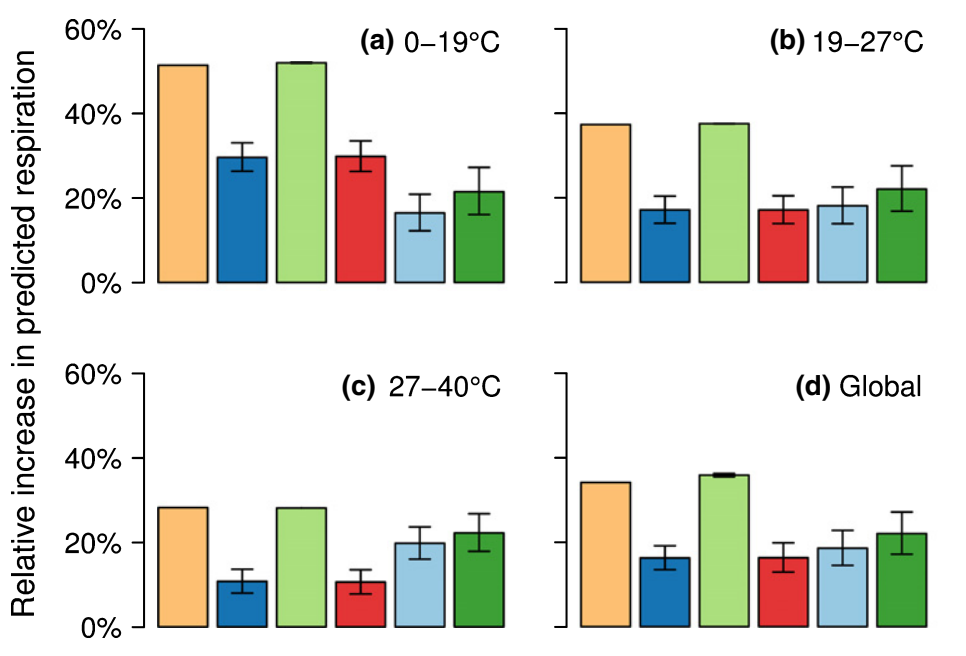

$\square$ Instant

Temporal

Spatial

$\square$ Both

Spatial-Equil

Both-Equil 
controlled by the shared acclimation parameter $r_{3}$ (as depicted graphically in Fig. 1b,d), and so it is not unexpected that this particular aspect of $R$ predictions is common to the Both and Temporal models.

Looking at spatial variation in mean annual respiration across the globe, models based on acclimation-over-time and variation-across-space all produced smaller increases in respiration with mean annual temperature than the model that excluded both of these effects (Instant, Fig. 3). Further, the rate at which respiration increased with mean annual temperature across global sites - the spatial equivalent of $Q_{10}$ (Piao et al., 2010) - was nearly the same between models incorporating acclimation-over-time (Temporal: $1.53 \quad(1.47-1.60$ given parameter uncertainty)) and variation-across-space (Spatial: 1.58 (1.52-1.65); Both: 1.53 (1.45-1.61)). The convergence of global temperature-dependent patterns of respiration between these models suggests that observed patterns in respiration at ambient temperatures (Atkin et al., 2015) might be explained by the experimentally measured strength of thermal acclimation in diverse plant taxa (Slot \& Kitajima, 2015), and may not necessarily be strongly related to longer term processes such as genetic adaptation or climate-related variation in species composition. Atkin et al. (2015) did not determine the relative importance of genotypic (i.e. inherent differences within and among species) and phenotypic variation (i.e. acclimation effects) in accounting for the observed global patterns.

Rates of leaf $R$ are controlled by respiratory capacity, demand for respiratory products, and the availability of respiratory substrate (i.e. carbohydrates). Some of these factors are influenced by genetic traits; for example, respiratory capacity and demand for respiratory products are related to leaf nitrogen $(\mathrm{N})$ and growth rate, respectively. Independent of species traits, acclimation is believed to operate through changes in leaf $\mathrm{N}$ and carbohydrates (Tjoelker et al., 2008) that are related to thermal history. Are geographical patterns in $R$ explained more by genetic factors or by thermal history, then? Common garden experiments in which warm- and cold-adapted populations of a species are grown together have frequently shown that local climatic conditions have a stronger influence on respiration than the location of source populations (Reich etal., 1996; Oleksyn etal., 1998; Gunderson etal., 2000; Lee etal., 2005; Tjoelker et al., 2008, 2009; Van de Weg et al., 2013). This general result implies that, within species, physiological acclimation exerts a greater degree of control over respiration processes than genetic adaptation. Among species, respiration tends to co-vary with leaf $\mathrm{N}$ (Wright et al., 2004, 2006; Atkin et al., 2015), but leaf $\mathrm{N}$ is only weakly associated with mean annual temperature and other climatic variables at a global scale (Reich \& Oleksyn, 2004; Wright et al., 2005). Species turnover across global temperature gradients is therefore unlikely to produce systematic differences in leaf respiration (at a common reference temperature) that could explain the observed climate-related variation in $R$. Taking these findings together with our current results, we may thus infer that physiological acclimation is largely responsible for temperaturedependent respiration patterns found across a global climate gradient.

\section{Responses to long-term temperature increases}

Our set of models describes three ways in which respiration rates may respond to climate warming at decadal to centennial timescales: two models (Instant and Spatial) assume that respiration will increase according to a temperature-dependent instantaneous response function, two others (Temporal and Both) assume that respiration increases will be determined by thermal acclimation, and a third pair (Spatial-Equil and Both-Equil) assume that future changes in respiration will mirror current patterns found across spatial climate gradients. Models based on an instantaneous response only (like many TBMs) produced the largest increases in future respiration (Fig.4). Because instantaneous responses are stronger at cold temperatures (under a temperaturedependent $Q_{10}$; Tjoelker et al., 2001), projected increases were largest in cold regions and smallest in warm ones. It is widely appreciated that the instantaneous response does not apply at time-scales longer than days to weeks, however (Atkin et al., 2000; Gifford, 2003; Dillaway \& Kruger, 2011). Accounting for thermal acclimation in our models reduced projected 110-yr increases in leaf-level respiration by approximately one-half. Qualitatively, this result is in line with those of studies that have incorporated thermal acclimation into TBMs and projected that acclimation will act to reduce carbon fluxes from terrestrial vegetation to the atmosphere under climate warming (Wythers et al., 2005, 2013; King et al., 2006; Chen \& Zhuang, 2013; Slot et al., 2014).

Interestingly, we find that spatial patterns in respiration at ambient temperatures (as described by the parameters $r_{1}$ and $r_{2}$ ) and measured acclimation effects $\left(r_{3}\right)$ each imply a similar global moderation of future warming-induced increases in leaf-level respiration. In the equilibrium models (Spatial-Equil and BothEquil in Fig. 4), relative increases in respiration with climate warming were about the same in cold (TWQ $<19^{\circ} \mathrm{C}$ ) and hot (TWQ $>27^{\circ} \mathrm{C}$ ) environments because the models assumed a positive linear relationship between (log-scale) respiration and TWQ. If this assumption holds true under future conditions, our results imply that leaf-level respiration increases in warm biomes, such as tropical forests and hot deserts, might not be offset to the degree expected from measurements of acclimation-over-time (Fig. 4c). Acclimation responses in tropical regions are not as well studied as in temperate ones, and thus have somewhat higher uncertainty (Table 2). Nevertheless, experimental warming treatments applied to eucalypt populations of tropical and temperate origins have recently shown that warm-origin populations can have stronger thermal acclimation responses than cool-origin populations because they down-regulate photosynthetic capacity (which is metabolically linked to respiration) as temperatures increase (Drake et al., 2015).

Comparative analyses with global respiration data sets (e.g. Wright etal., 2006; Piao et al., 2010) can be a useful means of assessing physiological acclimation, and may complement findings from experimental studies. Similar space-for-time substitutions have been applied in other areas of climate change biology, particularly in projecting how species' distributions track climatic niches (Guisan \& Thuiller, 2005). Caution is warranted, 
however, because models based on a space-for-time substitution may lack a sound biological basis if the processes governing changes in respiration do not keep pace with rates of climate change. However, as discussed above, it seems that physiological acclimation has considerable potential to moderate future increases in respiration even if species range shifts and genetic adaptation fail to keep pace with climate.

\section{Testing respiration models against data}

While the structure and parameter values for our models are well grounded in recently published data (Atkin et al., 2015; Slot \& Kitajima, 2015), it remains difficult to formally test the various models' predictions. Global databases of leaf traits such as TRY (Kattge etal., 2011) provide some of the most spatially and taxonomically extensive information on respiration, but this is generally restricted to snapshot measurements at a single time and temperature. Such data do not provide information on variation in respiration over longer periods, nor do they record site temperature histories that could be used to model acclimation responses to recent temperature changes. Annual autotrophic respiration data are also available from a global database of ecosystem-scale forest carbon balance, which combines eddy-covariance measurements with other observations (Luyssaert etal., 2007). These data have been useful in understanding controls over total annual respiration across the globe (Piao et al., 2010), but unfortunately they do not have the temporal resolution needed to examine seasonal acclimation processes. Networks of eddy- $\mathrm{CO}_{2}$ flux measurements (Baldocchi etal., 2001) provide measurements of net ecosystem exchange (NEE) with both high temporal resolution (continuous half-hourly respiration measurements over multiple years) and extensive spatial coverage (over 500 tower sites world-wide). In this case, nighttime $\mathrm{CO}_{2}$ fluxes provide a measure of ecosystem respiration, which is the sum of both autotrophic and heterotrophic respiration components. Eddy covariance measurements are often accompanied by measurements of soil respiration, which includes both heterotrophic respiration and below-ground autotrophic (root) respiration. However, soil respiration has different diel and seasonal patterns from ecosystem respiration (and by inference, aboveground respiration; Phillips et al., 2010), and so it is still difficult to evaluate thermal acclimation of leaf respiration with eddy covariance measurements.

Algorithms representing the response of respiration to temperature within TBMs need careful scrutiny (Smith \& Dukes, 2013). Without a mechanistic understanding for temperature-dependent $R$, the developers of TBMs have adopted simplistic model forms that do not necessarily hold up at large spatial and temporal scales. Vegetation modellers should always seek out ways to test the predictions of large-scale models, even if data sets such as those described above may be imperfect for this task. Here, we have shown that thermal acclimation effects from experimental studies are consistent with the best available data on global variation in respiration at ambient temperatures. These findings suggest there is considerable potential to improve our quantitative understanding of global carbon-climate dynamics by modifying respiration models to properly account for acclimation processes, and by ensuring that such models are well constrained by empirical data.

\section{Acknowledgements}

We thank participants in the $8^{\text {th }}$ New Phytologist Workshop on leaf respiration in large-scale vegetation models for valuable discussions that helped improve the final paper. We also thank three anonymous reviewers for their helpful comments. Funding for this project was provided by the National Science Foundation (NSF-IOS grant 1051789).

\section{References}

Aber JD, Federer CA. 1992. A generalized, lumped-parameter model of photosynthesis, evapotranspiration and net primary production in temperate and boreal forest ecosystems. Oecologia 92: 463-474.

Amthor JS, Baldocchi DD. 2001. Terrestrial higher plant respiration and net primary production. In: Roy J, Saugier B, Mooney HA, eds. Terrestrial global productivity. New York, NY, USA: Academic Press, 33-59.

Atkin OK, Atkinson LJ, Fisher RA, Campbell CD, Zaragoza-Castells J, Pitchford JW, Woodward FI, Hurry V. 2008. Using temperature-dependent changes in leaf scaling relationships to quantitatively account for thermal acclimation of respiration in a coupled global climate-vegetation model. Global Change Biology 14: 2709-2726.

Atkin OK, Bloomfield KJ, Reich PB, Tjoelker MG, Asner GP, Bonal D, Bönisch G, Bradford M, Cernusak LA, Cosio EG et al. 2015. Global variability in leaf respiration among plant functional types in relation to climate and leaf traits. New Phytologist 206: 614-636.

Atkin OK, Bruhn D, Tjoelker MG. 2005. Response of plant respiration to changes in temperature: mechanisms and consequences of variation in $\mathrm{Q}_{10}$ values and acclimation. In: Lambers $\mathrm{H}$, Ribas-Carbo $\mathrm{M}$, eds. Plant respiration. Dordrecht, the Netherlands: Springer, 95-135.

Atkin OK, Holly C, Ball MC. 2000. Acclimation of snow gum (Eucalyptus pauciflora) leaf respiration to seasonal and diurnal variations in temperature: the importance of changes in the capacity and temperature sensitivity of respiration. Plant, Cell \& Environment 23: 15-26.

Atkin OK, Meir P, Turnbull MH. 2014. Improving representation of leaf respiration in large-scale predictive climate-vegetation models. New Phytologist 202: 743-748.

Atkin OK, Tjoelker MG. 2003. Thermal acclimation and the dynamic response of plant respiration to temperature. Trends in Plant Science 8: 343-351.

Baldocchi D, Falge E, Gu L, Olson R, Hollinger D, Running S, Anthoni P, Bernhofer C, Davis K, Evans R et al. 2001. FLUXNET: a new tool to study the temporal and spatial variability of ecosystem-scale carbon dioxide, water vapor, and energy flux densities. Bulletin of the American Meteorological Society 82: 2415-2434.

Betts RA, Cox PM, Lee SE, Woodward FI. 1997. Contrasting physiological and structural vegetation feedbacks in climate change simulations. Nature 387: 796-799.

Bolstad PV, Reich P, Lee T. 2003. Rapid temperature acclimation of leaf respiration rates in Quercus alba and Quercus rubra. Tree Physiology 23: 969976.

Chen M, Zhuang Q. 2013. Modelling temperature acclimation effects on the carbon dynamics of forest ecosystems in the conterminous United States. Tellus Series B 65: 19156.

Collatz GJ, Ball JT, Grivet C, Berry JA. 1991. Physiological and environmental regulation of stomatal conductance, photosynthesis and transpiration: a model 
that includes a laminar boundary layer. Agricultural and Forest Meteorology 54: 107-136.

DeLucia EH, Drake JE, Thomas RB, Gonzalez-Meler M. 2007. Forest carbon use efficiency: is respiration a constant fraction of gross primary production? Global Change Biology 13: 1157-1167.

Dillaway DN, Kruger EL. 2011. Leaf respiratory acclimation to climate: comparisons among boreal and temperate tree species along a latitudinal transect. Tree Physiology 31: 1114-1127.

Drake JE, Aspinwall MJ, Pfautsch S, Rymer PD, Reich PB, Smith RA, Crous KY, Tissue DT, Ghannoum O, Tjoelker MG. 2015. The capacity to cope with climate warming declines from temperate to tropical latitudes in two widely distributed Eucalyptus species. Global Change Biology 21: 459472.

Friedlingstein P, Cox P, Betts R, Bopp L, von Bloh W, Brovkin V, Cadule P, Doney S, Eby M, Fung I et al. 2006. Climate-carbon cycle feedback analysis: results from the $\mathrm{C}^{4} \mathrm{MIP}$ model intercomparison. Journal of Climate 19: $3337-$ 3353.

Gifford RM. 2003. Plant respiration in productivity models: conceptualisation, representation and issues for global terrestrial carbon-cycle research. Functional Plant Biology 30: 171-186.

Gordon C, Cooper C, Senior CA, Banks H, Gregory JM, Johns TC, Mitchell JFB, Wood RA. 2000. The simulation of SST, seas ice extents and ocean heat transports in a version of the Hadley Centre coupled model without flux adjustments. Climate Dynamics 16: 147-168.

Guisan A, Thuiller W. 2005. Predicting species distribution: offering more than simple habitat models. Ecology Letters 8: 993-1009.

Gunderson CA, Norby RJ, Wullschleger SD. 2000. Acclimation of photosynthesis and respiration to simulated climatic warming in northern and southern populations of Acer saccharum: laboratory and field evidence. Tree Physiology 20: 87-96.

Hijmans RJ, Cameron SE, Parra JL, Jones PG, Jarvis A. 2005. Very high resolution interpolated climate surfaces for global land areas. International Journal of Climatology 25: 1965-1978.

IPCC. 2007. Climate Change 2007: the physical science basis. Contribution of working group I to the fourth assessment report of the Intergovernmental Panel on Climate Change. Solomon S, Qin D, Manning M, Chen Z, Marquis M, Averyt KB, Tignor M, Miller HL, eds. Cambridge, UK: Cambridge University Press, 996.

Kalnay E, Kanamitsu M, Kistler R, Collins W, Deaven D, Gandin L, Iredell M, Saha S, White G, Woollen J et al. 1996. The NCEP/NCAR 40-year reanalysis project. Bulletin of the American Meteorological Society 77: 437-471.

Kattge J, Diaz S, Lavorel S, Prentice IC, Leadley P, Bonisch G, Garnier E, Westoby M, Reich PB, Wright IJ et al. 2011. TRY - a global database of plant traits. Global Change Biology 17: 2905-2935.

King AW, Gunderson CA, Post WM, Weston DJ, Wullschleger SD. 2006. Plant respiration in a warmer world. Science 312: 536-537.

Lambers H, Chapin FS, Pons TL. 2008. Plant physiological ecology. New York, NY, USA: Springer.

Lee TD, Reich PB, Bolstad PV. 2005. Acclimation of leaf respiration to temperature is rapid and related to specific leaf area, soluble sugars and leaf nitrogen across three temperate deciduous tree species. Functional Ecology 19: 640-647.

Li S, Lü S, Zhang Y, Liu Y, Gao Y, Ao Y. 2014. The change of global terrestrial ecosystem net primary productivity (NPP) and its response to climate change in CMIP5. Theoretical and Applied Climatology. doi: 10.1007/s00704-014$1242-8$.

Loveys BR, Atkinson LJ, Sherlock DJ, Roberts RL, Fitter AH, Atkin OK. 2003. Thermal acclimation of leaf and root respiration: an investigation comparing inherently fast- and slow-growing plant species. Global Change Biology 9: 895910.

Luo Y. 2007. Terrestrial carbon-cycle feedback to climate warming. Annual Reviews in Ecology Evolution and Systematics 38: 683-712.

Luyssaert S, Inglima I, Jung M, Richardson AD, Reichstein M, Papale D, Piao SL, Schulze E-D, Wingate L, Matteucci G et al. 2007. $\mathrm{CO}_{2}$ balance of boreal, temperate, and tropical forests derived from a global database. Global Change Biology 13: 2509-2537.
Oleksyn J, Modrzynski J, Tjoelker MG, Zytkowiak Reich PB, Karolewski P. 1998. Growth and physiology of Picea abies populations from elevational transects: common garden evidence for altitudinal ecotypes and cold adaptation. Functional Ecology 12: 573-590.

Phillips SC, Varner RK, Frolking S, Munger JW, Bubier JL, Wofsy SC, Crill PM. 2010. Interannual, seasonal, and diel variation in soil respiration relative to ecosystem respiration at a wetland to upland slope at Harvard Forest. Journal of Geophysical Research: Biogeosciences 115: G02019.

Piao S, Luyssaert S, Ciais P, Janssens IA, Chen A, Cao C, Fang J, Friedlingstein P, Luo Y, Wang S. 2010. Forest annual carbon cost: a global-scale analysis of autotrophic respiration. Ecology 91: 652-661.

Raich JW, Rastetter EB, Melillo JM, Kicklighter DW, Steudler PA, Peterson BJ. 1991. Potential net primary productivity in South America: application of a global model. Ecological Applications 1: 399-429.

Reich PB, Oleksyn J. 2004. Global patterns of plant leaf N and P in relation to temperature and latitude. Proceedings of the National Academy of Sciences, USA 101: 11001-11006.

Reich PB, Oleksyn J, Tjoelker MG. 1996. Nitrogen respiration and nitrogen concentration in Scots Pine populations from a broad latitudinal range: a common garden test with field-grown trees. Functional Ecology 10: 768-776.

Reich PB, Walters MB, Ellsworth DS, Vose JM, Volin JC, Gresham C, Bowman WD. 1998. Relationships of leaf dark respiration to leaf nitrogen, specific leaf area and leaf life-span: a test across biomes and functional groups. Oecologia 114: 471-482.

Schimel DS, Participants VEMAP, Braswell BH. 1997. Continental scale variability in ecosystem processes: models, data, and the role of disturbance. Ecological Monographs 67: 251-271.

Slot M, Kitajima K. 2015. General patterns of acclimation of leaf respiration to warmer temperatures across biomes and plant types. Oecologia 177: 885900.

Slot M, Rey-Sánchez C, Gerber S, Lichstein JW, Winter K, Kitajima K. 2014. Thermal acclimation of leaf respiration of tropical trees and lianas: response to experimental canopy warming, and consequences for tropical forest carbon balance. Global Change Biology 20: 2915-2926.

Smith NG, Dukes JS. 2013. Plant respiration and photosynthesis in global-scale models: incorporating acclimation to temperature and $\mathrm{CO}_{2}$. Global Change Biology 19: 45-63.

Tjoelker MG, Oleksyn J, Lorenc-Plucinskia G, Reich PB. 2009. Acclimation of respiratory temperature responses in northern and southern populations of Pinus banksiana. New Phytologist 181: 218-229.

Tjoelker MG, Oleksyn J, Reich PB. 2001. Modelling respiration of vegetation: evidence for a general temperature-dependent $\mathrm{Q}_{10}$. Global Change Biology 7 : 223-230.

Tjoelker MG, Oleksyn J, Reich PB, Zytkowiak R. 2008. Coupling of respiration, nitrogen, and sugars underlies convergent temperature acclimation in Pinus banksiana across wide-ranging sites and populations. Global Change Biology 14: 782-797.

Van de Weg MJ, Fetcher N, Shaver G. 2013. Response of dark respiration to temperature in Eriophorum vaginatum from a 30-yearold transplant experiment in Alaska. Plant Ecology \& Diversity 6: 377-381.

Wright IJ, Reich PB, Atkin OK, Lusk CH, Tjoelker MG, Westoby M. 2006. Irradiance, temperature and rainfall influence leaf dark respiration in woody plants: evidence from comparisons across 20 sites. New Phytologist 169: 309319.

Wright IJ, Reich PB, Cornelissen JHC, Falster DS, Groom PK, Hikosaka K, Lee W, Lusk CH, Niinemets U, Oleksyn J et al. 2005. Modulation of leaf economic traits and trait relationships by climate. Global Ecology and Biogeography 14: 411-421.

Wright IJ, Reich PB, Westoby M, Acklerly DD, Baruch Z, Bongers F, Cavender-Bares J, Chapin FS, Cornelissen JHC, Diemer M et al. 2004. The world-wide leaf economics spectrum. Nature 428: 821-827.

Wythers KR, Reich PB, Bradford JB. 2013. Incorporating temperature-sensitive $\mathrm{Q}_{10}$ and foliar respiration acclimation algorithms modifies modeled ecosystem 
responses to global change. Journal of Geophysical Research: Biogeosciences 118: 77-90.

Wythers KR, Reich PB, Tjoelker MG, Bolstad PB. 2005. Foliar respiration acclimation to temperature and temperature variable $\mathrm{Q}_{10}$ alter ecosystem carbon balance. Global Change Biology 11: 435-449.
Yuan W, Luo Y, Li X, Liu S, Yu G, Zhou T, Bahn M, Black A, Desai A, Cescatti A et al. 2011. Redefinition and global estimation of basal ecosystem respiration rate. Global Biogeochemical Cycles 25: GB4002.

Zhang Y, Xu M, Chen H, Adams J. 2009. Global pattern of NPP to GPP ratio derived from MODIS data: effects of ecosystem type, geographical location and climate. Global Ecology and Biogeography 18: 280-290.

\section{About New Phytologist}

- New Phytologist is an electronic (online-only) journal owned by the New Phytologist Trust, a not-for-profit organization dedicated to the promotion of plant science, facilitating projects from symposia to free access for our Tansley reviews.

- Regular papers, Letters, Research reviews, Rapid reports and both Modelling/Theory and Methods papers are encouraged. We are committed to rapid processing, from online submission through to publication 'as ready' via Early View - our average time to decision is $<27$ days. There are no page or colour charges and a PDF version will be provided for each article.

- The journal is available online at Wiley Online Library. Visit www.newphytologist.com to search the articles and register for table of contents email alerts.

- If you have any questions, do get in touch with Central Office (np-centraloffice@lancaster.ac.uk) or, if it is more convenient, our USA Office (np-usaoffice@lancaster.ac.uk)

- For submission instructions, subscription and all the latest information visit www.newphytologist.com 